\title{
An Examination of the Mere Proximity Effect of Obesity Discrimination in Children in Chinese Culture*
}

\author{
Ai Chen, Shan Yin, Hui-Yi Tang, Zuo-Jun Wang \\ Ningbo University, Ningbo, China
}

\begin{abstract}
This study investigated the mere proximity effect of obesity discrimination in children in Chinese culture. One hundred and thirty children between 9 and 14 years old were asked to indicate how much they would like or to be friends with a target character, who was either male or female and average-weight or overweight. The target character was presented with four other background characters of the same gender, whom were either average-weight or overweight. The findings demonstrated that female targets were preferred less when presented with overweight background characters than when presented with average-weight background characters. However, male targets were preferred more when presented with overweight background characters than when presented with average-weight background characters. The findings indicated an anti-fat mere proximity effect in female children but a contrary effect in male children in Chinese culture. These findings may have implications for a better understanding of weight bias from a cultural perspective.
\end{abstract}

Keywords: obesity discrimination, mere proximity effect, children, Chinese culture

\section{Introduction}

Social stigma towards obese youths is pervasive and can have serious consequences for both emotional and physical health of these obese children and adolescents (e.g., Puhl \& Latner, 2007). Some research has shown that the stigmatization of obesity would generalize to those who have, or not have, a relationship with a stigmatized individual (Hebl \& Mannix, 2003; Neuberg, Smith, Hoffman, \& Russell, 1994; Penny \& Haddock, 2007a, 2007b). An interesting study by Hebl and Mannix (2003) revealed that an average-weight male job applicant was assessed negatively when he was perceived as sitting next to an overweight female stranger, as much as he was perceived as sitting next to his overweight girlfriend. The phenomenon that obesity stigma would generalize to average-weight individuals who was proximity to the obese individual was termed the mere proximity effect (Hebl \& Mannix, 2003).

Given that close proximity is very important in children socialization and forming friends, Penny and Haddock (2007a) examined whether the mere proximity effect of obesity would occur in children. In their study, a target character who was either male or female and either average-weight or overweight with four other

\footnotetext{
*Acknowledgments: The National Natural Science Foundation of China (No. 31200791), the Humanity and Social Science Youth foundation of Ministry of Education of China (Grant No. 12YJC190029) and the Zhejiang Provincial Natural Science Foundation of China (Grant No. LQ12G01001) supported this paper.

Ai Chen, B.S., Department of Psychology, Ningbo University.

Shan Yin, B.S., Department of Psychology, Ningbo University.

Hui-Yi Tang, B.S., Department of Psychology, Ningbo University.

Zuo-Jun Wang, Ph.D., Department of Psychology, Ningbo University.
} 
background characters of the same gender, all of whom were either average-weight or overweight, was presented to 5-10 years old children. The participants were asked to indicate whether they would like to be friends with a target character. The results showed that overall, overweight targets were liked significantly less than average-weight targets, and in line with the mere proximity effect that average-weight female targets were liked significantly less when they were presented with overweight background characters. Hence, mere proximity effect was obtained among 5-10 years old children.

Culture is known to be an interesting and important factor that may impact obesity stigma. Latner, Stunkard, and Wilson (2005) found that Asian participants liked obese individuals more than white participants, and African-American female participants liked obese individuals more than white male or female participants. Crystal, Watanabe, and Chen (2000) found that 5th grade Japanese children had a more positive attitude toward overweight characters than American children.

Chinese culture seems especially obesity-friendly. Lee, Ho, and Hsu (1993) suggested that the Chinese have regarded plumpness as desirable and attractive. Some Chinese sayings, such as "Xin-kuan-ti-pan (心宽体 胖)" and “Fu-tai (富态)" are all positive descriptions of fat or obesity. Hao et al. (2010) found that more than $40 \%$ of the 3rd-4th grade primary school students viewed obese children as friendly, liking to make friends, self-confident, and having good appetite, though they were considered lazy, inflexible, having bad figure, and so on. Ma, Li, Han, Ren, and Xu (2004) found that about 30\% senior middle school students viewed obese companions as kind, gentle, happy, friendly, sociable, strong, with good appetite, and so on, while only $20 \%$ of the students viewed obese companions as lazy, careless, puffy, slow-acting, and so on. Chinese parents apparently believe that obese children are healthier and obesity reflects their love for their children (Bush, 2003).

Another characteristic of Chinese culture related to social stigma is its hackneyed idea of preferring boys to girls (e.g., Parish \& Whyte, 1980), which makes females more prone to be stigmatized than males. Accordingly, the obesity-friendly attitude in Chinese culture may be of differential concern to male children and female children. For instance, Ma et al. (2004) found that male high school students viewed obese females as more prone to be bullied and difficulty in further employment, while female high school students viewed obese males as strong, happy, with good appetite, and so on.

The present study aimed to examine the mere proximity effect of obesity discrimination in children in Chinese culture. We assumed that the mere proximity anti-fat discrimination effect may not necessarily be observed in children in Chinese culture, especially in male children. The present study aimed to test this assumption.

\section{Experiment}

\section{Method}

Participants. One hundred and thirty 9-14 years old primary school students took part in this research. Of these 130 students, 25 were excluded from analyses because they did not complete the whole experiment. The final sample included 64 males and 41 females with average age at 10.88. Given that some previous researches has demonstrated that age would moderate the obesity discrimination (Latner, Stunkard, \& Wilson, 2005; Powlishta, Serbin, Doyle, \& White, 1994), we divided the participants into three age groups, leading to 56 $(53.3 \%)$ falling in 9-10 years old, 31 (29.5\%) falling in 11-12 years old and $18(17.1 \%)$ falling in 13-14 years old. 
Materials. The materials were prepared and characterized as described by Penny and Haddock (2007b). Ten characters ( 5 females, 5 males), each one of which was a colored cartoon drawing of a child, were designed. Both an average-weight version and an overweight version were designed for each character, which were identical expect for their weight (see Figure 1). Thus, each individual character was either male or female, overweight or average-weight, and was placed on a separated card with $10 \mathrm{~cm}$ by $15 \mathrm{~cm}$.

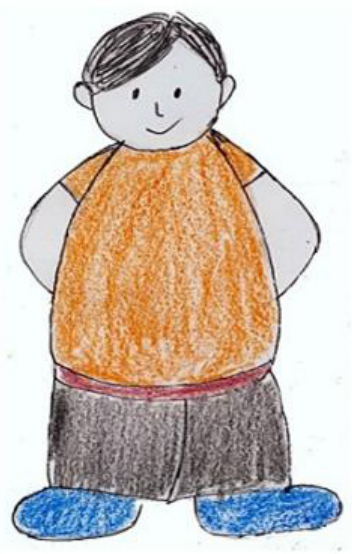

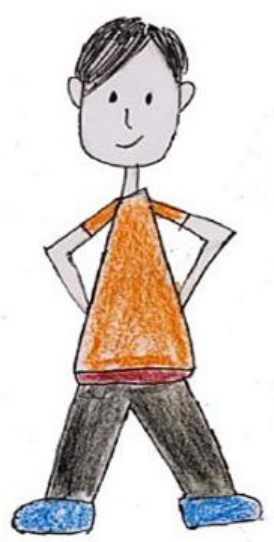
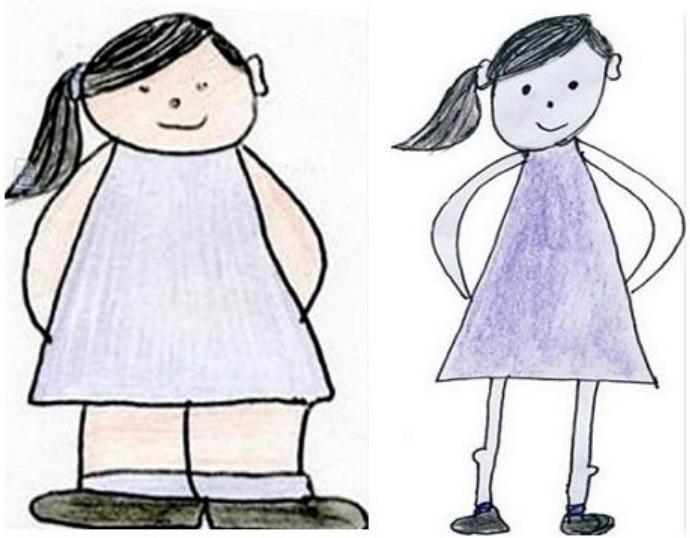

Figure 1. Characters.

\section{Procedure and Design}

The study was conducted in classroom. The experimenter told participants that she was interested in how much children would like or to be friends with other children. The participants were told that they would see a picture of a person and asked: (1) how much they would like this individual; and (2) how much they would like to be friends with this individual. The response options available were "Extremely not", "Probably not", "Yes" or "Extremely yes" (scored from 1 to 4, with 1 indicating "Extremely not" and 4 "Extremely yes"). The target character was presented in the middle of four other background characters of the same gender, all of whom were either average-weight or overweight. The participants were told to ignore the background characters and only make judgments on the target character in the middle.

A 2 (participant gender: male and female) $\times 3$ (age group: 9-10, 11-12, 13-14) $\times 2$ (target gender: male and female $) \times 2$ (target size: overweight and average-weight $) \times 2$ (background size: overweight and average-weight $)$ mixed-subject design was employed in the experiment. Participant gender and age group (9-10, 11-12, 13-14) were manipulated between-participant, while the last three variables were manipulated within-participant: gender of the target and background character (both male or female), target size, and the size of the background characters. Each participant was asked to response to 16 tasks, with 2 counterparts of each 8 combinations outlined above.

\section{Results}

We collapsed the two measures of "being liked" and "being friends" into one dependent measure of "preference". A 2 (participant gender) $\times 2$ (target gender) $\times 2$ (target size) $\times 2$ (background size) repeated measures analysis of variance was employed. The results revealed no significant main effects of participant gender, age, target gender or background size were observed $\left(p_{\mathrm{s}}>0.05\right)$. The results also revealed a significant main effect of target size on measure of preference $\left(F_{(1,99)}=66.082, p<0.001\right)$ and a significant two-way interaction between participant gender and target gender $\left(F_{(1,99)}=85.691, p<0.001\right)$. 
As the most important concern of the present study, we observed a three-way interaction between target size, background size, and target gender $\left(F_{(1,99)}=1.674, p<0.01\right)$. This interaction is further dissected as a function of target gender. The results revealed that, for male targets, the interaction between target size and background size was significant $(p<0.05$ ) (see Table 1). However, interestingly, male targets (especially average-weight male targets) were rated higher when presented with overweight background characters than with average-weight background characters $\left(p_{s}<0.05\right)$. This finding indicated that male children were preferred more when they were connected to overweight children than with average-weight children, which was contrary to the mere proximity anti-fat discrimination effect.

Table 1

Ratings of Male Target as a Function of Target Size and Background Size

\begin{tabular}{llc}
\hline & \multicolumn{2}{c}{ Target size } \\
\cline { 2 - 3 } & Average-weight & Overweight \\
\hline Background size & & \\
Average-weight & 2.509 & 2.173 \\
Overweight & 2.956 & 2.325 \\
\hline
\end{tabular}

For female targets, the interaction between target size and background size was not significant $(p=0.372)$ (see Table 2). Further analysis demonstrated that average-weight targets were more preferred than overweight targets either when they were presented with average-weight or overweight background characters $\left(p_{s}<0.001\right)$. Furthermore, both average-weight and overweight female targets were more preferred when were presented with average-weight background characters than with overweight background characters, indicating a mere proximity effect in female children $\left(p_{s}<0.001\right)$.

Table 2

Ratings of Female Target as a Function of Target Size and Background Size

\begin{tabular}{llc}
\hline & \multicolumn{2}{c}{ Target size } \\
\cline { 2 - 3 } & Average-weight & Overweight \\
\hline Background size & & \\
Average-weight & 3.075 & 2.373 \\
Overweight & 2.654 & 2.023 \\
\hline
\end{tabular}

\section{Discussion and Conclusion}

The present study demonstrated that male children were preferred more when they were presented with overweight background characters than when they were presented with average-weight background characters, indicating a reversed pattern of the mere proximity anti-fat discrimination effect in male children in Chinese culture. However, overweight female children were preferred less when presented with overweight background characters than with average-weight background characters, indicating a mere proximity anti-fat discrimination effect.

The findings of the present study may have implications for a better understanding of weight bias from a cultural perspective. The findings in the present study illustrated that the findings regarding the social stigma towards obese youths found in the western culture may not necessarily extended to Chinese culture. Further more, as we have found in the present study, even in the same culture, social stigma may differ across demographic groups such as gender. The findings also may have implications for social and educational policy in China. As we have suggested, Chinese culture is traditional of preference of boys to girls. Girls in China fare 
worse than boys in many dimensions: They obtain less schooling, have lower labor force participation, earn lower salaries, are more likely to be poor, and often lack fundamental rights (Duflo, 2005). One often-cited extreme manifestation of this phenomenon is that mortality rates are substantially higher for girls than for boys (S. H. Chen, Y. C. Chen, \& Liu, 2010). All of these lead us to conclude that we should give girls more concern and love in China. Our study especially had a better guiding significance for caring obese girls.

Some previous research indicated that not only their playmates will exhibit bias toward obese children, but also these negative attitudes had translated into discrimination toward obese children in a number of people, including the colleagues, medical and health care workers, teachers even parents. Crandall $(1991 ; 1995)$ found that parents provide less college financial support for their heavy weight daughters compared to their average-weight daughters and heavyweight or average-weight sons. Teachman and Brownell (2001) suggested that even health care specialists had strong negative associations toward obese persons. The present study only examined the attitudes of companions toward obese children in Chinese culture. Future work could examine the attitudes of adults toward male or female obese children in Chinese culture.

\section{References}

Bush, K. R. (2003). Commentary: Physical and mental health of contemporary Chinese children. Journal of Family and Economic Issues, 24, 397-401.

Chen, S. H., Chen, Y. C., \& Liu, J. T. (2010). The effect of sibling sex composition on child mortality and education. Mimeo: Royal Holloway University of London.

Cossrow, N. H. F., Jeffery, R. W., \& McGuire, M. T. (2001). Understanding weight stigmatization: A focus group study. Journal of Nutrition Education, 33, 208-214.

Crystal, D. S., Watanabe, H., \& Chen, R. S. (2000). Preference for diversity in competitive and cooperative contexts: A study of American and Japanese children and adolescents. International Journal of Behavioral Development, 24(3), 348-355.

Crandall, C. S. (1991). Do heavy-weight students have more difficulty paying for college? Personality and Social Psychology Bulletin, 17, 606-611.

Crandall, C. S. (1995). Do parents discriminate against their heavyweight daughters? Personality and Social Psychology Bulletin, $21,724-735$.

Duflo, E. (2005). Gender equality in development. MIT Working Paper.

Gortmaker, S. L., Must, A., Perrin, J. M., Sobol, A. M., \& Dietz, W. H. (1993). Social and economic consequences of overweight in adolescence and young adulthood. N. Engl. J. Med, 329, 1008-1012.

Hao, L. N., Li, Y. P., Du, S. M., Hu, X. Q., Yang, W., Ma, G. S., et al. (2010). Perception and attitude on obese children of urban primary school students in Beijing. Chinese Journal of School Health, 31(2), 161-163.

Hebl, M. R., \& Mannix, L. M. (2003). The weight of obesity in evaluating others: A mere proximity effect. Personality and Social Psychology Bulletin, 29(1), 28-38.

Kirsten Krahnstoever Davison \& Leann Lipps Birch. (2004). Predictors of Fat Stereotypes among 9-year-old girls and their parents. Obesity Research, 12(1), 86-94.

Latner, J. D., Stunkard, A. J., \& Wilson, T. G. (2005). Stigmatized students: Age, sex, and ethnicity effects in the stigmatization of obesity. Obesity Research, 13, 1226-1231.

Lee, S., Ho, T. P., \& Hsu, L. K. G. (1993). Fat phobic and non-fat phobic anorexia nervosa: A comparative study of 70 Chinese patients in Hong Kong. Psychological Medicine, 23, 999-1017.

Ma, G. S., Li, Y. P., Han, X. M., Ren, H., \& Xu, Y. J. (2004). The perception and attitudes on obese adolescents of Huiwen high school students in Beijing. Chinese Center for Disease Control and Prevention, 20(11), 975-978.

Marsh, H. W., Hau, K. T., Sung, R. Y. T., \& Yu, C. W. (2007). Childhood obesity, gender, actual-ideal body image discrepancies, and physical self-concept in Hong Kong children: Cultural differences in the value of moderation. Developmental Psychology, 43(3), 647-662.

Neuberg, S. L., Smith, D. M., Hoffman, J. C., \& Russell, F. J. (1994). When we observe stigmatized and "normal" individuals interacting: Stigma by association. Personality and Social Psychology Bulletin, 20, 196-209. 
Parish, W. L., \& Whyte, M. K. (1980). Village and family in contemporary China. University of Chicago Press.

Penny, H., \& Haddock, G. (2007a). Anti-fat prejudice among children: The "mere proximity" effect in 5-10 year olds. Journal of Experimental Social Psychology, 43, 678-683.

Penny, H., \& Haddock, G. (2007b). Children's stereotypes of overweight children. British Journal of Developmental Psychology, $25,409-418$.

Puhl, R. M., \& Latner, J. D. (2007). Stigma, obesity, and the health of the nation's children. Psychological Bulletin, 133(4), 557-580.

Powlishta, K. K., Serbin, L. A., Doyle, A., \& White, D. R. (1994). Gender, ethnic, and body type biases: The generality of prejudice in childhood. Developmental Psychology, 30, 526-536.

Teachman, B. A., \& Brownell, K. D. (2001). Implicit anti-fat bias among health professional: us anyone immune? International Journal of Obesity, 25, 1525-1531. 